\title{
28 Research Square \\ Relationship between fibrinogen/albumin ratio and early renal damage markers in patients with hypertension
}

jiankai jian dong ( $\square$ 582402973@qq.com )

qinghai univeisity https://orcid.org/0000-0003-0659-9328

Yaping Zhang

Qinghai Provincial People's Hospital

\section{Research Article}

Keywords: Fibrinogen/albumin ratio, Inflammation, Hypertension, Renal damage

Posted Date: September 27th, 2021

DOl: https://doi.org/10.21203/rs.3.rs-843155/v1

License: (c) (1) This work is licensed under a Creative Commons Attribution 4.0 International License.

Read Full License 


\section{Abstract}

Aim:To investigate the relationship between fibrinogen/albumin ratio (FAR) and early renal damage in hypertensive patients.

Patients \& methods:A retrospective study included 626 patients with hypertension, grouped according to the FAR tertiles and the presence or absence of early renal impairment. Early renal damage indicators[Serum cystatin $c$ and $\beta 2$ microglobulin] were detected in each group, and the differences between groups were compared, and the factors affecting early renal damage indicators were analyzed.

Results:Serum cystatin c(CysC) and $\beta 2$ microglobulin( $\beta 2-M G)$ levels in patients increased with FAR. In the renal impairment group, the fibrinogen and FAR were significantly increased, and serum albumin was decreased. FAR was positively correlated with $\beta 2-M G$ and CysC. Regression analysis showed that FAR level was a factor affecting blood $\beta 2-M G(\beta=6.632, p \otimes 0.001)$ and $\operatorname{Cys} C(\beta=1.991, p \llbracket 0.001)$.

Conclusion:Elevated FAR is an independent risk factor for early renal damage in hypertensive patients

\section{Introduction}

Hypertension is the most common chronic disease in clinic [1], and also the most important risk factor for cardiovascular and cerebrovascular diseases. Its main complications, such as coronary heart disease, heart failure, and stroke, not only cause disability and have a high mortality rate, but also severely consume medical and social resources. Thus it can be seen that hypertension has become an important public health problem worldwide. The kidney is one of the important target organs for damage caused by hypertension. The early clinical symptoms of hypertensive nephropathy are not significant, but usually they are only manifested as increased blood pressure, which brings difficulties to clinical diagnosis and treatment [2]. With the development of the disease, patients successively show abnormal changes such as increased serum creatinine(Scr) and blood urea nitrogen(BUN), microalbuminuria(mALB) and persistent urine protein, finally causing chronic renal failure or uremia. At this time, the kidney damage is often irreversible [3]. Blood $\beta 2$-microglobulin ( $\beta 2-M G$ )and serum cystatin $C(C y s C)$ are sensitive and reliable indicators reflecting early renal damage, and significantly superior to serum creatinine, urea nitrogen and other markers of renal injury $[4,5]$.

Low-grade inflammation is an important factor to cause and maintain elevated blood pressure, and the levels of circulating inflammatory factors are closely related to the degree of hypertension and target organ damage [6-8]. Elevated inflammatory markers, including neutrophils [9], tumor necrosis factor-a [10] and fibrinogen [11], are common in patients with hypertension. As a marker of inflammation in the acute phase, Fibrinogen is associated with hypertension [12] and diabetes [13], and has an important pathophysiological role in aggravating the progression of kidney disease [14]. Albumin is an important factor involved in systemic inflammatory response, and its decreased serum level can enhance the inflammatory response $[15,16]$. Epidemiological evidence suggests that low serum albumin levels are 
associated with many cardiovascular diseases, such as coronary heart disease, hypertension and heart failure $[17,18]$.

Therefore, we have found a new inflammation-based risk index, fibrinogen to albumin ratio (FAR). The study has confirmed the correlation between this new index of FAR and many diseases such as cancer [19], end-stage renal disease [20] and coronary artery disease [21], with strong clinical value. However, there are few studies on the relationship between FAR and early renal impairment markers. The purpose of this study was to explore the relationship between FAR and early renal impairment indicators in patients with hypertension.

\section{Patients \& Methods}

\subsection{Study Population}

A total of 636 inpatients with primary hypertension from June 2018 to February 2021 in Qinghai Provincial People's Hospital were included as the subjects. According to the FAR tertiles, patients were divided into low (L-FAR) group (FAR $<0.057, n=212)$, medium (M-FAR) group $(0.057 \leq F A R \leq 0.07, n=$ $214)$ and high $(H-F A R)$ group $(F A R>0.07, n=210) .636$ patients were also divided into normal renal function group (eGFR $\geq 90 \mathrm{ml} / \mathrm{min} / 1.73 \mathrm{~m}^{2}, \mathrm{n}=453$ ) and impaired renal function group (eGFR: $60-89$ $\mathrm{mL} / \mathrm{min} / 1.73 \mathrm{~m}^{2}, \mathrm{n}=183$ ) according to eGFR. The study protocol was approved by Ethics Committee of Qinghai Provincial People's Hospital. All participants signed the informed consent.

\subsection{Inclusion and exclusion criteria}

The inclusion criteria are as follows:: $\nabla$ Age $\geq 18$ years old, hypertension grade $1-3$, no gender limitation, complete clinical data. खPrimary hypertension is diagnosed by referring to 2017 American College of Cardiology and American Heart Association Blood Pressure Guidelines. Hypertension was defined as having two independent blood pressure measurements $\geq 140 / 90 \mathrm{mmHg}$ or having received antihypertensive drug treatment. 『eGFR $\geq 60 \mathrm{ml} / \mathrm{min} / 1.73 \mathrm{~m}^{2} \square$

The exclusion criteria are as follows: $₫$ various acute and chronic infectious diseases; $\otimes$ Patients with chronic hepatitis, cirrhosis of liver, liver cancer, liver failure and other liver diseases in the past, or those who have taken drugs that affect liver function in the near future; $\otimes$ Patients with primary glomerular disease, renal artery stenosis, and severe renal impairment; $\forall$ Complicated with chronic consumptive diseases such as malignant tumor, autoimmune disease, hematological system disease, thyroid disease, and tuberculosis; $\otimes$ Secondary hypertension, coronary heart disease, diabetes, acute cardiovascular and cerebrovascular diseases;

\subsection{Data Collection}

Clinical data such as gender, age, body mass, smoking history, drinking history and hypertension course were recorded accurately, and body massindex (BMI) was calculated. 
$\mathrm{BMI}=$ body mass $(\mathrm{kg}) /$ height $(\mathrm{m}) 2$.

All subjects were fasted for 10-12 hours one day before the experiment, and 4-5 ml venous blood was taken on an empty stomach in the early morning of the next day, and the serum was collected after centrifugation at $4000 \mathrm{r} / \mathrm{min}$. Blood biochemical tests were performed with Beckman AU5800 automatic biochemical analyzer, and the biochemical indexes such as serum albumin(Alb), urea nitrogen(BUN), serum creatinine(Scr), total cholesterol(TC), high density lipoprotein cholesterol (HDL-C), low density lipoprotein cholesterol (LDL-C), Serum uric acid(SUA) and blood $\beta 2$ microglobulin(B2-MG) were measured. Serum fibrinogen(Fib) level was detected by coagulation method with Sysmex CS5100 automatic coagulation analyzer. The levels of fibrinogen and albumin were collected, and FAR was calculated as the ratio of fibrinogen to albumin. MDRD formula was used to calculate and estimate the

glomerular filtration rate (eGFR)[22]: male: eGFR $=186 \times \mathrm{Scr}(\mathrm{mg} / \mathrm{dl})^{-1.154} \times$ age $(\text { years })^{-0.203}$; Female: eGFR $=186 \times \operatorname{Scr}(\mathrm{mg} / \mathrm{dl})^{-1.154} \times$ age $(\text { years })^{-0.203 \times 0.742}$.

\subsection{Statistical Analysis}

Statistical analysis was performed using SPSS 26.0 (SPSS Inc.).Measurement data conforming to normal distribution were expressed as mean \pm standard deviation. A Student's t-test was used for comparison between two groups, one-way analysis of variance was used for comparison among multiple groups, and q test was used for pairwise comparison between groups. The measurement data of nonnormal distribution was expressed as M(P25, P75). The Kruskal-Wallis rank sum test was used for comparison among multiple groups, and Mann-whitney $U$ test was used for pairwise comparison. Enumeration data were compared using $\mathrm{X}^{2}$ test; Pearson correlation analysis and Spearman rank correlation analysis were used for correlation analysis. Multifactor linear stepwise regression was used to analyze the influencing factors of serum $\beta 2$ - microglobulin and cystatin $\mathrm{C}$. The level of statistical significance for all the above tests was defined at a probability value of less than $0.05(P<0.05)$.

\section{Results}

A total of 626 patients (mean age $58.98 \pm 12.22$ years, 360 men) with hypertension were included in the study.The baseline clinical characteristics and laboratory measurements of patients within the groups are presented in Table 1. Participants were grouped into tertiles, according to their FAR level.A significant positive association between age and FAR level was identified (Table 1). The mean age in the lower group of FAR was $54.50 \pm 11.12$, which was significantly lower than the medium (59.68 \pm 12.17$)$ and high group $(62.79 \pm 11.92)(P \otimes 0.001)$. The levels of Male, Smoking history, Drinking history, Hypertension grade, Duration of hypertension, SBP, DBP, BMI, TC, HDL-C, Scr, SUA, BUN and mALB in L-FAR group, M-FAR group and H-FAR group had no significant difference $(p>0.05)$ The levels of Fib, LDL-C, Alb, CysC, $\beta 2-M G$ and eGFR in L-FAR group, M-FAR group and $H$-FAR group were significantly different $(P<0.05)$. Compared with L-FAR group, Fib, CysC and $\beta 2-M G$ were higher in M-FAR group and H-FAR group, while Alb was lower. LDL-C was higher and EGFR was lower in M-FAR group. Compared with M-FAR group, Fib and $\beta 2-$ $M G$ in H-FAR group were higher, while Alb was lower $(P<0.05$,Fig. 1$) \rrbracket$ 
Table 1

:Clinical and Laboratory Characteristics According to the FAR Tertile

\begin{tabular}{|c|c|c|c|c|c|}
\hline & $\begin{array}{l}\text { L-FAR group } n \\
=212\end{array}$ & $\begin{array}{l}\text { M-FAR group } \\
n=214\end{array}$ & $\begin{array}{l}\text { H-FAR group } \\
n=210\end{array}$ & $F / X^{2} / H$ & $\mathbf{P}$ \\
\hline Age(years) & $54.50 \pm 11.12$ & $59.68 \pm 12.17 *$ & $\begin{array}{l}62.79 \pm \\
11.92^{\star \star}\end{array}$ & 26.809 & $\varangle 0.001$ \\
\hline male[n(\%)] & $133(62 \%)$ & $113(52 \%)$ & $114(53 \%)$ & 4.963 & 0.084 \\
\hline $\begin{array}{l}\text { Smoking history } \\
{[\mathrm{n}(\%)]}\end{array}$ & $59(27 \%)$ & $51(23 \%)$ & $51(24 \%)$ & 1.076 & 0.584 \\
\hline $\begin{array}{l}\text { Drinking history } \\
{[n(\%)]}\end{array}$ & $50(23 \%)$ & $42(19 \%)$ & $41(19 \%)$ & 1.374 & 0.503 \\
\hline $\begin{array}{l}\text { Hypertension } 3 \\
\text { grade[n(\%)] }\end{array}$ & 198(93\%) & 204(95\%) & 202(96\%) & 1.811 & 0.404 \\
\hline $\begin{array}{l}\text { Duration of } \\
\text { hypertension(months) }\end{array}$ & $70.47 \pm 70.07$ & $77.27 \pm 74.02$ & $87.54 \pm 78.19$ & 2.832 & 0.060 \\
\hline $\mathrm{SBP}(\mathrm{mmHg})$ & $135.81 \pm 12.63$ & $135.41 \pm 14.44$ & $136.55 \pm 13.91$ & 0.379 & 0.685 \\
\hline $\mathrm{DBP}(\mathrm{mmHg})$ & $82.83 \pm 12.28$ & $80.57 \pm 10.92$ & $80.71 \pm 10.07$ & 2.736 & 0.066 \\
\hline $\operatorname{BMI}\left(\mathrm{kg} / \mathrm{m}^{2}\right)$ & $25.64 \pm 3.63$ & $25.20 \pm 3.72$ & $25.67 \pm 4.03$ & 0.995 & 0.370 \\
\hline $\mathrm{Fib}(\mathrm{g} / \mathrm{L})$ & $2.02 \pm 0.31$ & $2.57 \pm 0.23^{\star}$ & $3.31 \pm 0.61^{\star * *}$ & 506.661 & $\nabla 0.001$ \\
\hline $\mathrm{TC}(\mathrm{mmol} / \mathrm{l})$ & $4.16 \pm 0.90$ & $4.69 \pm 4.18$ & $4,24 \pm 0.84$ & 2.77 & 0.063 \\
\hline $\mathrm{HDL}-\mathrm{C}(\mathrm{mmol} / \mathrm{L})$ & $1.01 \pm 0.21$ & $1.05 \pm 0.24$ & $1.02 \pm 0.23$ & 1.699 & 0.184 \\
\hline LDL-C(mmol/L) & $2.48 \pm 0.68$ & $2.64 \pm 0.75^{\star}$ & $2.61 \pm 0.68$ & 3.129 & 0.044 \\
\hline $\mathrm{Alb}(\mathrm{g} / \mathrm{L})$ & $41.61 \pm 4.61$ & $40.51 \pm 3.32^{\star}$ & $38.05 \pm 3.76^{\star \star}$ & 45.191 & $\nabla 0.001$ \\
\hline CysC(mg/L) & $0.96 \pm 0.26$ & $1.04 \pm 0.31^{*}$ & $1.07 \pm 0.29^{*}$ & 8.685 & $\nabla 0.001$ \\
\hline $\operatorname{Scr}(\mu \mathrm{mol} / \mathrm{L})$ & $68.88 \pm 14.24$ & $69.61 \pm 14.75$ & $68.31 \pm 15.64$ & 0.408 & 0.665 \\
\hline ß2-MG(mg/L) & $1.77 \pm 0.59$ & $1.95 \pm 0.66^{\star}$ & $2.09 \pm 0.74^{\star \star}$ & 12.583 & $\otimes 0.001$ \\
\hline \multicolumn{6}{|c|}{ 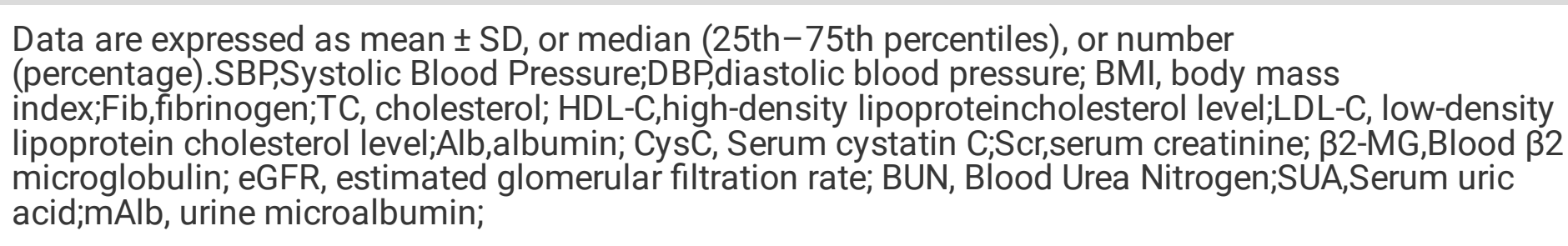 } \\
\hline \multicolumn{6}{|c|}{${ }^{\star} \mathrm{p}<0.05$ compared with L-FAR group; ${ }^{* \star} \mathrm{p}<0.01$ compared with M-FAR group } \\
\hline . & & & & & \\
\hline
\end{tabular}




\begin{tabular}{|c|c|c|c|c|c|}
\hline & $\begin{array}{l}\text { L-FAR group } n \\
=212\end{array}$ & $\begin{array}{l}\text { M-FAR group } \\
n=214\end{array}$ & $\begin{array}{l}\text { H-FAR group } \\
n=210\end{array}$ & $\mathrm{~F} / \mathrm{X}^{2} / \mathrm{H}$ & $\mathbf{P}$ \\
\hline $\begin{array}{l}\text { eGFR[ml.min }{ }^{-1} \text {. } \\
\left.\left(1.73 \mathrm{~m}^{2}\right)^{-1}\right]\end{array}$ & $103.29 \pm 19.89$ & $97.87 \pm 21.17 *$ & $99.69 \pm 21.03$ & 3.820 & 0.022 \\
\hline $\operatorname{SUA}(\mu \mathrm{mol} / \mathrm{L})$ & $\begin{array}{l}383.78 \pm \\
111.20\end{array}$ & $\begin{array}{l}376.52 \pm \\
113.91\end{array}$ & $\begin{array}{l}367.11 \pm \\
124.40\end{array}$ & 1.042 & 0.353 \\
\hline $\mathrm{BUN}(\mathrm{mmol} / \mathrm{L})$ & $5.44 \pm 1.45$ & $5.69 \pm 1.55$ & $5.74 \pm 1.83$ & 2.036 & 0.131 \\
\hline $\mathrm{mALB}(\mathrm{mg} / \mathrm{L})$ & $1.00(0.59,1.84)$ & $1.05(0.60,2.06)$ & $1.17(0.56,3.34)$ & 3.729 & 0.155 \\
\hline \multicolumn{6}{|c|}{ 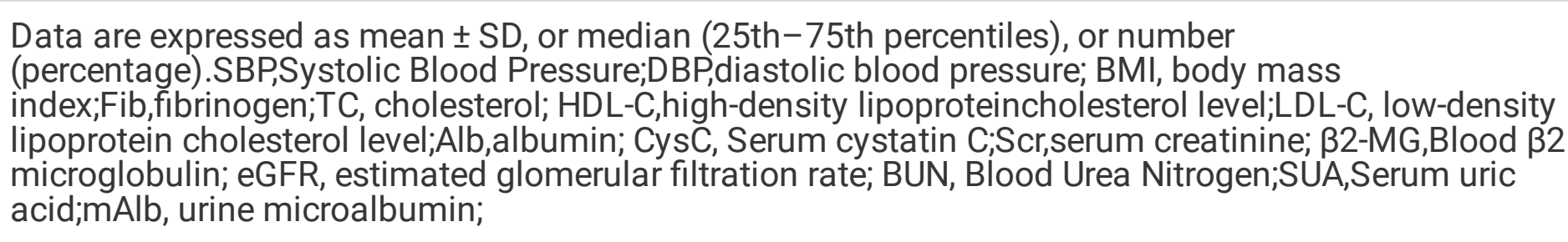 } \\
\hline \multicolumn{6}{|c|}{${ }^{*} \mathrm{p}<0.05$ compared with L-FAR group; ${ }^{* *} \mathrm{p}<0.01$ compared with M-FAR group } \\
\hline
\end{tabular}

Table 2 shows the comparison of patient data according to eGFR levels (Normal renal function group: eGFR $\geq 90 \mathrm{ml} / \mathrm{min} / 1.73 \mathrm{~m} 2, \mathrm{n}=453$; Renal impairment group: eGFR: $60-89 \mathrm{~mL} / \mathrm{min} / 1.73 \mathrm{~m} 2, \mathrm{n}=183$ ). There was no significant difference in the levels of Male, Hypertension grade, Smoking history, Drinking history, SBP, DBP, BMI, LDL-C, TC and HDL-C between the two groups $(P>0.05)$. The levels of Age, Duration of hypertension, Fib, FAR, SUA, BUN, blood $32-\mathrm{MG}, \mathrm{CysC}$, Scr and mAlb in the renal impairment group were significantly higher than those in the normal renal function group. The levels of ALB and eGFR were significantly lower than those in the normal renal function group, and the differences were statistically significant $(P<0.05$,Table 2,Fig. 2$)$. 
Table 2

Baseline clinical and laboratory characteristics in the normal and impaired renal function groups

\begin{tabular}{|c|c|c|c|c|}
\hline & $\begin{array}{l}\text { Normal renal function } \\
\text { group } \\
n=453\end{array}$ & $\begin{array}{l}\text { Renal impairment group } \\
n=183\end{array}$ & $t / z$ & $\mathbf{P}$ \\
\hline Age(years) & $57.11 \pm 11.92$ & $63.62 \pm 11.73$ & 6.264 & $\bowtie 0.001$ \\
\hline male[n(\%)] & $250(55 \%)$ & $110(60 \%)$ & 1.285 & 0.257 \\
\hline Hypertension 3 grade[n(\%)] & $430(94 \%)$ & $174(95 \%)$ & 0.007 & 0.934 \\
\hline $\begin{array}{l}\text { Smoking history } \\
{[\mathrm{n}(\%)]}\end{array}$ & $113(24 \%)$ & $48(26 \%)$ & 0.114 & 0.736 \\
\hline $\begin{array}{l}\text { Drinking history } \\
\text { [n(\%)] }\end{array}$ & $94(20 \%)$ & $39(21 \%)$ & 0.025 & 0.875 \\
\hline $\begin{array}{l}\text { Duration of } \\
\text { hypertension(months) }\end{array}$ & $97.57 \pm 87.10$ & $70.65 \pm 67.13$ & 3.755 & $\varangle 0.001$ \\
\hline $\mathrm{SBP}(\mathrm{mmHg})$ & $136.14 \pm 13.12$ & $135.36 \pm 14.95$ & -0.658 & 0.511 \\
\hline DBP(mmHg) & $81.90 \pm 11.41$ & $80.06 \pm 10.44$ & -1.882 & 0.060 \\
\hline $\operatorname{BMI}\left(\mathrm{kg} / \mathrm{m}^{2}\right)$ & $25.44 \pm 3.79$ & $25.65 \pm 3.82$ & 0.634 & 0.526 \\
\hline $\mathrm{HDL}-\mathrm{C}(\mathrm{mmol} / \mathrm{L})$ & $1.04 \pm 0.22$ & $1.00 \pm 0.24$ & -1.674 & 0.095 \\
\hline $\mathrm{TC}(\mathrm{mmol} / \mathrm{l})$ & $4.25 \pm 0.89$ & $4.65 \pm 4.51$ & 1.209 & 0.228 \\
\hline LDL-C(mmol/L) & $2.57 \pm 0.72$ & $2.59 \pm 0.66$ & 0.365 & 0.715 \\
\hline $\mathrm{Fib}(\mathrm{g} / \mathrm{L})$ & $2.59 \pm 0.65$ & $2.71 \pm 0.72$ & 2.029 & 0.043 \\
\hline $\mathrm{Alb}(\mathrm{g} / \mathrm{L})$ & $40.28 \pm 4.28$ & $39.54 \pm 3.96$ & -2.03 & 0.043 \\
\hline FAR & $0.06 \pm 0.01$ & $0.07 \pm 0.02$ & 2.372 & 0.018 \\
\hline SUA( $\mu \mathrm{mol} / \mathrm{L})$ & $352.40 \pm 106.59$ & $433.44 \pm 120.38$ & 8.356 & $\bowtie 0.001$ \\
\hline BUN(mmol/L) & $5.33 \pm 1.47$ & $6.35 \pm 1.73$ & 7.043 & $\otimes 0.001$ \\
\hline$\beta 2-\mathrm{MG}(\mathrm{mg} / \mathrm{L})$ & $1.76 \pm 0.55$ & $2.36 \pm 0.76$ & 9.764 & $\varangle 0.001$ \\
\hline CysC(mg/L) & $0.94 \pm 0.24$ & $1.22 \pm 0.32$ & 10.61 & $₫ 0.001$ \\
\hline $\operatorname{Scr}(\mu \mathrm{mol} / \mathrm{L})$ & $62.84 \pm 11.00$ & $84.03 \pm 12.18$ & 21.318 & $\triangle 0.001$ \\
\hline
\end{tabular}

Data are expressed as mean \pm SD, or median (25th-75th percentiles), or number (percentage).FAR,fibrinogen/albumin ratio. 


\begin{tabular}{|c|c|c|c|c|}
\hline & $\begin{array}{l}\text { Normal renal function } \\
\text { group } \\
n=453\end{array}$ & $\begin{array}{l}\text { Renal impairment group } \\
n=183\end{array}$ & $t / z$ & $P$ \\
\hline $\begin{array}{l}\text { eGFR[ml.min } \\
\left.\left(1.73 \mathrm{~m}^{2}\right)^{-1}\right]\end{array}$ & $109.69 \pm 16.47$ & $77.00 \pm 8.27$ & -33.16 & $\nabla 0.001$ \\
\hline $\mathrm{mALB}(\mathrm{mg} / \mathrm{L})$ & $1.00(0.56,2.00)$ & $1.36(0.66,3.68)$ & -3.132 & 0.002 \\
\hline
\end{tabular}

The results of correlation analysis showed that there was a negative correlation between FAR level and Alb in patients with hypertension $(P<0.05)$. They were positively correlated with Age, Duration of hypertension, LDL-C, Fib, blood $\beta 2-M G, C y s C$ and mALB $(p<0.05)$. There is no correlation between FAR level and SBP, DBP, BMI, TC, HDL-C, Scr, BUN, eGFR and SUA. After adjusting Sex and Age, FAR was still positively correlated with $\beta 2-\mathrm{MG}$, CysC and mALB in blood ( $p>0.05$, Table 3$)$. 
Table 3

Correlation analysis between FAR level and various indicators in patients with hypertension

\begin{tabular}{|c|c|c|c|c|}
\hline & \multicolumn{2}{|c|}{ No calibration } & \multicolumn{2}{|c|}{ Calibration age, sex } \\
\hline & $r\left(r_{s}\right)$ & $\mathrm{p}$ & $r\left(r_{s}\right)$ & $\mathrm{p}$ \\
\hline Age(years) & 0.221 & $\varangle 0.001$ & - & - \\
\hline Duration of hypertension(months) & 0.080 & 0.044 & 0.004 & 0.922 \\
\hline $\mathrm{SBP}(\mathrm{mmHg})$ & 0.072 & 0.071 & 0.082 & 0.040 \\
\hline $\mathrm{DBP}(\mathrm{mmHg})$ & -0.004 & 0.928 & 0.063 & 0.115 \\
\hline $\mathrm{BMI}\left(\mathrm{kg} / \mathrm{m}^{2}\right)$ & 0.032 & 0.418 & 0.057 & 0.151 \\
\hline $\mathrm{TC}(\mathrm{mmol} / \mathrm{l})$ & 0.007 & 0.856 & 0.018 & 0.645 \\
\hline $\mathrm{HDL}-\mathrm{C}(\mathrm{mmol} / \mathrm{L})$ & 0.001 & 0.985 & -0.035 & 0.375 \\
\hline LDL-C(mmol/L) & 0.099 & 0.012 & 0.103 & 0.01 \\
\hline $\mathrm{Fib}(\mathrm{g} / \mathrm{L})$ & 0.928 & $\varangle 0.001$ & 0.930 & $₫ 0.001$ \\
\hline $\operatorname{Alb}(g / L)$ & -0.458 & $\varangle 0.001$ & -0.422 & $\otimes 0.001$ \\
\hline CysC(mg/L) & 0.206 & $\varangle 0.001$ & 0.148 & $\otimes 0.001$ \\
\hline$\beta 2-M G(m g / L)$ & 0.269 & $\varangle 0.001$ & 0.204 & $\otimes 0.001$ \\
\hline $\operatorname{Scr}(\mu \mathrm{mol} / \mathrm{L})$ & 0.007 & 0.854 & 0.028 & 0.475 \\
\hline $\mathrm{BUN}(\mathrm{mmol} / \mathrm{L})$ & 0.068 & 0.088 & 0.041 & 0.297 \\
\hline eGFR[ml.min $\left.{ }^{-1} \cdot\left(1.73 \mathrm{~m}^{2}\right)^{-1}\right]$ & -0.064 & 0.105 & -0.012 & 0.758 \\
\hline $\mathrm{SUA}(\mu \mathrm{mol} / \mathrm{L})$ & -0.003 & 0.945 & 0.020 & 0.607 \\
\hline mALB(mg/L) & 0.239 & $\varangle 0.001$ & 0.247 & $\bowtie 0.001$ \\
\hline
\end{tabular}

The relationship between inflammatory markers and early renal injury indicators was analyzed by multiple linear regression with blood $\beta 2-M G$ and serum cystatin $C$ as dependent variables and age, disease course month and FAR level as independent variables. The results showed that FAR level was the influencing factor of blood $\beta 2-\mathrm{MG}(\beta=6.632, \mathrm{p} \otimes 0.001)$ and serum cystatin $C(\beta=1.991, \mathrm{p} \otimes 0.001)($ Table 4). 
Table 4

Multivariate linear regression analysis of far and $\beta 2-\mathrm{MG}$ and CysC

\begin{tabular}{|c|c|c|c|c|c|c|}
\hline & & $\beta$ & SE & Beta & $\mathrm{t}$ & $\mathbf{p}$ \\
\hline \multirow[t]{4}{*}{$\beta 2-M G$} & constant & 0.400 & 0.132 & & 3.023 & 0.003 \\
\hline & FAR & 6.362 & 1.218 & 0.192 & 5.225 & $\otimes 0.001$ \\
\hline & Age & 0.018 & 0.002 & 0.327 & 8.377 & $\otimes 0.001$ \\
\hline & Duration of hypertension & 0.001 & $₫ 0.001$ & 0.061 & 1.606 & 0.109 \\
\hline \multirow[t]{4}{*}{ CysC } & constant & 0.454 & 0.058 & & 7.765 & $\bigotimes 0.001$ \\
\hline & FAR & 1.991 & 0.538 & 0.134 & 3.552 & $\otimes 0.001$ \\
\hline & Age & 0.007 & 0.001 & 0.296 & 7.406 & $\otimes 0.001$ \\
\hline & Duration of hypertension & $\otimes 0.001$ & $₫ 0.001$ & 0.085 & 2.184 & 0.029 \\
\hline
\end{tabular}

\section{Discussion}

The mechanism of renal damage caused by hypertension has not been elucidated, including hemodynamic and non-hemodynamic mechanisms. The involvement of immune inflammatory response in the pathogenesis of hypertensive renal damage has gradually become a hot research topic in recent years [23]. The main purpose of this study was to investigate the effect of FAR, an inflammatory marker, on early renal damage in patients with hypertension. Samples consisted of patients with essential hypertension, grouped according to the FAR tertiles and the presence or absence of early renal impairment. The levels of early renal impairment indexes and inflammatory markers were compared among different groups. The results showed that the serum CysC and $\beta 2-M G$ levels of patients in the MFAR group and H-FAR group were higher than those in the L-FAR group. The levels of fibrin and FAR in the renal impairment group were significantly higher than those in the normal renal function group. The serum albumin level was significantly lower than that in the normal renal function group. The FAR level had a positive correlation with $\beta 2-M G$ and serum CysC. The results of multiple linear regression analysis showed that: FAR level is a factor affecting blood $\beta 2-M G$ and serum CysC, suggesting that for patients with hypertension and increased levels of inflammatory factors, the early renal damage was more significant. FAR level is related to the changes of blood $\beta 2-M G$ and serum CysC.

Hypertension is a low-grade inflammatory state disease, and inflammation plays an important role in the occurrence, development and complications of hypertension [24]. Persistent inflammation is also a key feature of CKD, which can lead to progressive renal fibrosis, endothelial dysfunction and other aggravating renal damage [25]. Fibrinogen, a precursor of fibrin, is produced by the liver and has a serum concentration of $2.0-4.0 \mathrm{~g} / \mathrm{L}$, which plays an important role in thrombosis and inflammatory reaction 
[26]. A large number of studies have found that serum fibrinogen levels are related to cardiovascular risk factors or cardiovascular events [27], and fibrinogen has also been found to be significantly associated with renal impairment. For example, Lin et al. [28] in a cross-sectional study of 732 T2DM men found that serum fibrinogen was associated with decreased glomerular filtration rate, and another study reported [14] that elevated serum fibrinogen levels in T2DM patients were associated with end-stage renal disease. Fibrinogen has been identified as an independent risk factor for death and cardiovascular events in patients with chronic renal disease Stage 3 and 4 [29]. Celik et al. [30] found that serum fibrinogen levels were independently associated with the risk of contrast-induced acute kidney injury in patients with acute coronary syndrome receiving coronary intervention (OR 1.006,95\% $\mathrm{Cl} 1.003-1.009, \mathrm{p}<0.001)$. In summary, chronic inflammatory conditions, reflected in elevated serum fibrinogen levels, may have exacerbated renal impairment. This study found a significant increase in plasma fibrinogen levels in patients with early renal impairment compared to patients with normal renal function, demonstrating that mean concentrations in patients with renal impairment were significantly higher than in patients with normal renal function.

Serum albumin is a synthetic protein of the liver that maintains the plasma colloid osmotic pressure, transports lipids, and participates in the processes of acute and chronic inflammation [15, 31]. Serum albumin concentration is related to the increase of inflammatory load in vivo, and inflammation is related to the decrease of albumin synthesis and increase of catabolism [16]. Studies have shown that serum albumin level is a useful and reliable prognostic indicator for acute kidney injury [32]. An independent predictor of acute kidney injury in patients after $\mathrm{PCl}$ for acute coronary syndrome when serum albumin reduction at admission was identified [33]. The mechanism of kidney injury caused by low serum albumin is considered to be multi-factorial, including inhibition of platelet aggregation, increase of blood viscosity, reduction of antioxidant capacity, inflammatory response and destruction of endothelial function $[16,17,34]$. This study found that serum albumin levels decreased with the increase in inflammatory index levels, and serum albumin levels in patients with renal impairment were lower than those in the normal renal function group, which was consistent with the results of the above study.

It has been recently found that FAR is a novel inflammatory indicator that combines fibrinogen and albumin, and can more sensitively reflect the inflammatory state. Many studies have revealed the relationship between FAR and many diseases, including cardiovascular diseases. For example, that study by Mahmut et al. [35] indicate that FAR is a powerful predictor of early morning blood pressure increase in newly diagnosed and treated patients with primary hypertension and may be a bet predictor than single fibrinogen and albumin. In addition, FAR is significantly associated with the severity and short-term prognosis of coronary artery stenosis in patients with st-segment elevation myocardial infarction [36, 37]. Notably, a recent study found that FAR was independently associated with the occurrence of PC-Aki (postcontrast acute kidney disease) [38]. Jing JuanYang et al. [32] revealed that preoperative serum fibrinogen was associated with the risk of acute kidney injury after heart valve replacement $(O R=1.212,95 \% \mathrm{Cl}$ $1.1089-1.347, P=0.003)$. 
At present, the markers used for evaluating renal impairment mainly include Scr, BUN, CysC, $\beta 2-M G$, etc. Among them, $\mathrm{CysC}$, which is used to reflect glomerular function, is an effective indicator for the assessment of glomerular filtration rate at present. With high sensitivity and strong specificity, it is less affected by factors such as gender, age, and muscle content, and is one of the ideal indicators for the assessment of early renal injury [39]. Ozer et al. [40] found that compared with traditional renal injury markers Scr and BUN, CysC is the best indicator for predicting renal function in primary hypertension patients with mild decreased GFR. $\beta 2-M G$, a small molecular protein, has a low but constant content in the body, which is a sensitive indicator for assessing renal tubular function. In the early stage of decreased glomerular filtration function, serum $\beta 2$-MG level can be increased, and increases with the aggravation of renal impairment. It is a sensitive indicator reflecting early lesions of hypertensive renal injury [41]. In this study, the relationship between the new inflammatory marker FAR and early renal impairment was explored for the first time. The results showed that as the level of FAR increased, the levels of $\beta 2-M G$ and CysC also increased, and there was a significant positive correlation between them. FAR level was a factor affecting blood $\beta 2-M G$ and serum CysC. With the increase of the value of FAR, the levels of $\beta 2-M G$ and CysC increased by 6.362 and 1.991 respectively. It indicated that inflammation was a risk factor for early renal injury in patients with hypertension, and monitoring of FAR could well assess early renal impairment.

\section{Limitations}

Of course, the study has some limitations. First, our study results are based on a retrospective study design, and selection bias is inevitable. Second, this study was conducted in Chinese patients with hypertension, and no normal subjects were recruited as the control group. The results need to be verified in a healthy population and other ethnic groups. Third, the majority of the subjects included in the study with hypertension were in Grade 3 hypertension, and the proportion of other subjects was small, which limited the intensity of the results and conclusions of this study. Finally, we only measured albumin, fibrinogen, and FAR levels at admission; we did not perform continuous measurements of these parameters.

\section{Conclusion\& future perspective}

This study has shown that elevated FAR levels are an independent risk factor for early renal damage in hypertensive patients and baseline FAR can provide a useful indicator of early renal damage in hypertensive patients and is superior to established inflammatory markers. Therefore, for patients with hypertension, in order to prevent target organ damage, we should pay attention to the treatment of inflammation while strictly controlling the blood pressure to reach the standard, in order to delay the occurrence of renal damage and reduce the risk of cardiovascular and cerebrovascular complications.

\section{Declarations}

Author Contributions: JD: Data collection, analyses and interpretation, and writing of the final manuscript. JD and YZ: Study design, Data interpretation and revising manuscript. The author(s) read and approved 
the final manuscript.

Acknowledgements: None.

Funding $\square$ There is no funding support.

Conflicts of Interest $\otimes T$ he authors declare that there are no conflicts of interest.

Information pertaining to writing assistance: N/A

Ethical disclosure: The authors state that they have obtained appropriate institutional review board approval or have followed the principles outlined in the Declaration of Helsinki for all human or animal experimental investigations. In addition, for investigations involving human subjects, informed consent has been obtained from the participants involved.

Data sharing statement: The data that support the findings of this study are available from the corresponding author upon reasonable request.

\section{References}

1. Mills K, Bundy J, Kelly T et al (2016) Global Disparities of Hypertension Prevalence and Control: A Systematic Analysis of Population-Based Studies From 90 Countries. Circulation 134(6):441-450. doi:10.1161/circulationaha.115.018912

2. Seccia T, Caroccia B, Calò L (2017) Hypertensive nephropathy. Moving from classic to emerging pathogenetic mechanisms. Journal of hypertension 35(2):205-212.

doi:10.1097/hjh.0000000000001170

3. Griffin K. Hypertensive Kidney Injury and the Progression of Chronic Kidney Disease. Hypertension (Dallas, Tex. 1979). 2017;70(4):687-694. doi:10.1161/hypertensionaha.117.08314

4. Meng L, Yang Y, Qi L, Wang X, Xu G, Zhang B (2012) Elevated serum cystatin C is an independent predictor of cardiovascular events in people with relatively normal renal function. $J$ Nephrol 25(3):426-430. doi:10.5301/jn.5000020

5. Shirzai A, Yildiz N, Biyikli N, Ustunsoy S, Benzer M, Alpay H (2014) Is microalbuminuria a risk factor for hypertension in children with solitary kidney? Pediatric nephrology (Berlin. Germany) 29(2):283288. doi:10.1007/s00467-013-2641-2

6. Rodriguez-Iturbe B, Pons H, Johnson R (2017) Role of the Immune System in Hypertension. Physiological reviews 97(3):1127-1164. doi:10.1152/physrev.00031.2016

7. Guzik T, Touyz R. Oxidative Stress, Inflammation, and Vascular Aging in Hypertension. Hypertension (Dallas, Tex. 1979). 2017;70(4):660-667. doi:10.1161/hypertensionaha.117.07802 
8. Barrows I, Ramezani A, Raj D. Inflammation, Immunity, and Oxidative Stress in Hypertension-Partners in Crime? Advances in chronic kidney disease. 2019;26(2):122-130. doi:10.1053/j.ackd.2019.03.001

9. Liu X, Zhang Q, Wu H et al (2015) Blood Neutrophil to Lymphocyte Ratio as a Predictor of Hypertension. Am J Hypertens 28(11):1339-1346. doi:10.1093/ajh/hpv034

10. Silveira-Nunes G, Durso D Jr (2020) L, et al. Hypertension Is Associated With Intestinal Microbiota Dysbiosis and Inflammation in a Brazilian Population. Front Pharmacol 11:258. doi:10.3389/fphar.2020.00258

11. Jae S, Kurl S, Laukkanen J et al (2015) Relation of C-reactive protein, fibrinogen, and cardiorespiratory fitness to risk of systemic hypertension in men. The American journal of cardiology 115(12):1714-1719. doi:10.1016/j.amjcard.2015.03.016

12. Davalos D, Akassoglou K (2012) Fibrinogen as a key regulator of inflammation in disease. Semin Immunopathol 34(1):43-62. doi:10.1007/s00281-011-0290-8

13. Bembde A (2012) A study of plasma fibrinogen level in type-2 diabetes mellitus and its relation to glycemic control. Indian journal of hematology blood transfusion: an official journal of Indian Society of Hematology Blood Transfusion 28(2):105-108. doi:10.1007/s12288-011-0116-9

14. Zhang J, Wang Y, Zhang R et al (2018) Serum fibrinogen predicts diabetic ESRD in patients with type 2 diabetes mellitus. Diabetes Res Clin Pract 141:1-9. doi:10.1016/j.diabres.2018.04.025

15. Eckart A, Struja T, Kutz A et al (2020) Relationship of Nutritional Status, Inflammation, and Serum Albumin Levels During Acute Illness: A Prospective Study. Am J Med 133(6):713-722.e7. doi:10.1016/j.amjmed.2019.10.031

16. Don B, Kaysen G (2004) Serum albumin: relationship to inflammation and nutrition. Seminars in dialysis 17(6):432-437. doi:10.1111/j.0894-0959.2004.17603.x

17. Arques $S$ (2018) Human serum albumin in cardiovascular diseases. European journal of internal medicine 52:8-12. doi:10.1016/j.ejim.2018.04.014

18. Oda E (2014) Decreased serum albumin predicts hypertension in a Japanese health screening population. Intern Med (Tokyo Japan) 53(7):655-660. doi:10.2169/internalmedicine.53.1894

19. Sun $D, A n L$, Lv G (2020) Albumin-fibrinogen ratio and fibrinogen-prealbumin ratio as promising prognostic markers for cancers: an updated meta-analysis. World J Surg Oncol 18(1):9. doi:10.1186/s12957-020-1786-2

20. Zou Y, Zhu Z, Zhou J et al. Fibrinogen/Albumin ratio: A more powerful prognostic index for patients with end-stage renal disease. Eur J Clin Invest. 2020:e13266. doi:10.1111/eci.13266

21. Zhang D, Mao X, Wu T et al (2020) The Fibrinogen-to-Albumin Ratio Is Associated With Outcomes in Patients With Coronary Artery Disease Who Underwent Percutaneous Coronary Intervention. Clinical applied thrombosis/hemostasis: official journal of the International Academy of Clinical Applied Thrombosis/Hemostasis 26:1076029620933008. doi:10.1177/1076029620933008

22. Ma Y, Zuo L, Chen J et al (2006) Modified glomerular filtration rate estimating equation for Chinese patients with chronic kidney disease. Journal of the American Society of Nephrology: JASN 17(10):2937-2944. doi:10.1681/asn.2006040368 
23. McMaster W, Kirabo A, Madhur M, Harrison D (2015) Inflammation, immunity, and hypertensive endorgan damage. Circulation research 116(6):1022-1033. doi:10.1161/circresaha.116.303697

24. Kim K, Lee J, Chang $\mathrm{H}$ et al (2008) Association between blood pressure variability and inflammatory marker in hypertensive patients. Circulation journal: official journal of the Japanese Circulation Society 72(2):293-298. doi:10.1253/circj.72.293

25. Ruiz-Ortega M, Rayego-Mateos S, Lamas S, Ortiz A, Rodrigues-Diez R (2020) Targeting the progression of chronic kidney disease. Nature reviews Nephrology 16(5):269-288. doi:10.1038/s41581-019-0248-y

26. Sörensen I, Susnik N, Inhester T et al (2011) Fibrinogen, acting as a mitogen for tubulointerstitial fibroblasts, promotes renal fibrosis. Kidney international 80(10):1035-1044. doi:10.1038/ki.2011.214

27. Stec J, Silbershatz H, Tofler $\mathrm{G}$ et al (2000) Association of fibrinogen with cardiovascular risk factors and cardiovascular disease in the Framingham Offspring Population. Circulation 102(14):16341638. doi:10.1161/01.cir.102.14.1634

28. Lin J, Hu F, Rimm E, Rifai N, Curhan G (2006) The association of serum lipids and inflammatory biomarkers with renal function in men with type II diabetes mellitus. Kidney international 69(2):336342. doi:10.1038/sj.ki.5000021

29. Weiner $D$, Tighiouart $H$, Elsayed $E$ et al (2008) The relationship between nontraditional risk factors and outcomes in individuals with stage 3 to 4 CKD. American journal of kidney diseases: the official journal of the National Kidney Foundation 51(2):212-223. doi:10.1053/j.ajkd.2007.10.035

30. Celik I, Kurtul A, Duran M et al (2016) Elevated serum fibrinogen levels and risk of contrast-induced acute kidney injury in patients undergoing a percutaneous coronary intervention for the treatment of acute coronary syndrome. Coron Artery Dis 27(1):13-18. doi:10.1097/mca.0000000000000295

31. Quinlan G, Martin G, Evans T (2005) Albumin: biochemical properties and therapeutic potential. Hepatology 41(6):1211-1219. doi:10.1002/hep.20720

32. Li N, Qiao H, Guo J et al (2019) Preoperative hypoalbuminemia was associated with acute kidney injury in high-risk patients following non-cardiac surgery: a retrospective cohort study. BMC anesthesiology 19(1):171. doi:10.1186/s12871-019-0842-3

33. Murat S, Kurtul A, Yarlioglues M (2015) Impact of Serum Albumin Levels on Contrast-Induced Acute Kidney Injury in Patients With Acute Coronary Syndromes Treated With Percutaneous Coronary Intervention. Angiology 66(8):732-737. doi:10.1177/0003319714551979

34. Kawai Y, Masutani K, Torisu K et al (2018) Association between serum albumin level and incidence of end-stage renal disease in patients with Immunoglobulin A nephropathy: A possible role of albumin as an antioxidant agent. PloS one 13(5):e0196655. doi:10.1371/journal.pone.0196655

35. Ozdemir M, Yurtdas M, Asoglu R, Yildirim T, Aladag N, Asoglu E (2020) Fibrinogen to albumin ratio as a powerful predictor of the exaggerated morning blood pressure surge in newly diagnosed treatmentnaive hypertensive patients. Clin Exp Hypertens Nov 16(8):692-699. doi:10.1080/10641963.2020.1779282 42 ). 
36. Zhao Y, Yang J, Ji Y et al (2019) Usefulness of fibrinogen-to-albumin ratio to predict no-reflow and short-term prognosis in patients with ST-segment elevation myocardial infarction undergoing primary percutaneous coronary intervention. Heart vessels 34(10):1600-1607. doi:10.1007/s00380-01901399-w

37. Karahan O, Acet $\mathrm{H}$, Ertaş $\mathrm{F}$ et al (2016) The relationship between fibrinogen to albumin ratio and severity of coronary artery disease in patients with STEMI. Am J Emerg Med 34(6):1037-1042. doi:10.1016/j.ajem.2016.03.003

38. Wang C, Li G, Liang X et al (2020) Predictive Value of Fibrinogen-to-Albumin Ratio for Post-Contrast Acute Kidney Injury in Patients Undergoing Elective Percutaneous Coronary Intervention. Med Sci Monit Jul 20:26:e924498. doi:10.12659/MSM.924498

39. Woo K, Choi J, Kim B, Kim J, Han J (2014) Clinical usefulness of serum cystatin C as a marker of renal function. Diabetes metabolism journal 38(4):278-284. doi:10.4093/dmj.2014.38.4.278

40. Ozer B, Dursun B, Baykal A, Gultekin M, Suleymanlar G (2005) Can cystatin C be a better marker for the early detection of renal damage in primary hypertensive patients? Ren Fail 27(3):247-253. doi:10.1081/jdi-56635

41. Cao J, Hou R, Lu J et al (2019) The predictive value of $\beta 2-M G$ and TGF- $\beta$ for elderly hypertensive nephropathy. Experimental therapeutic medicine 17(4):3065-3070. doi:10.3892/etm.2019.7278

\section{Figures}




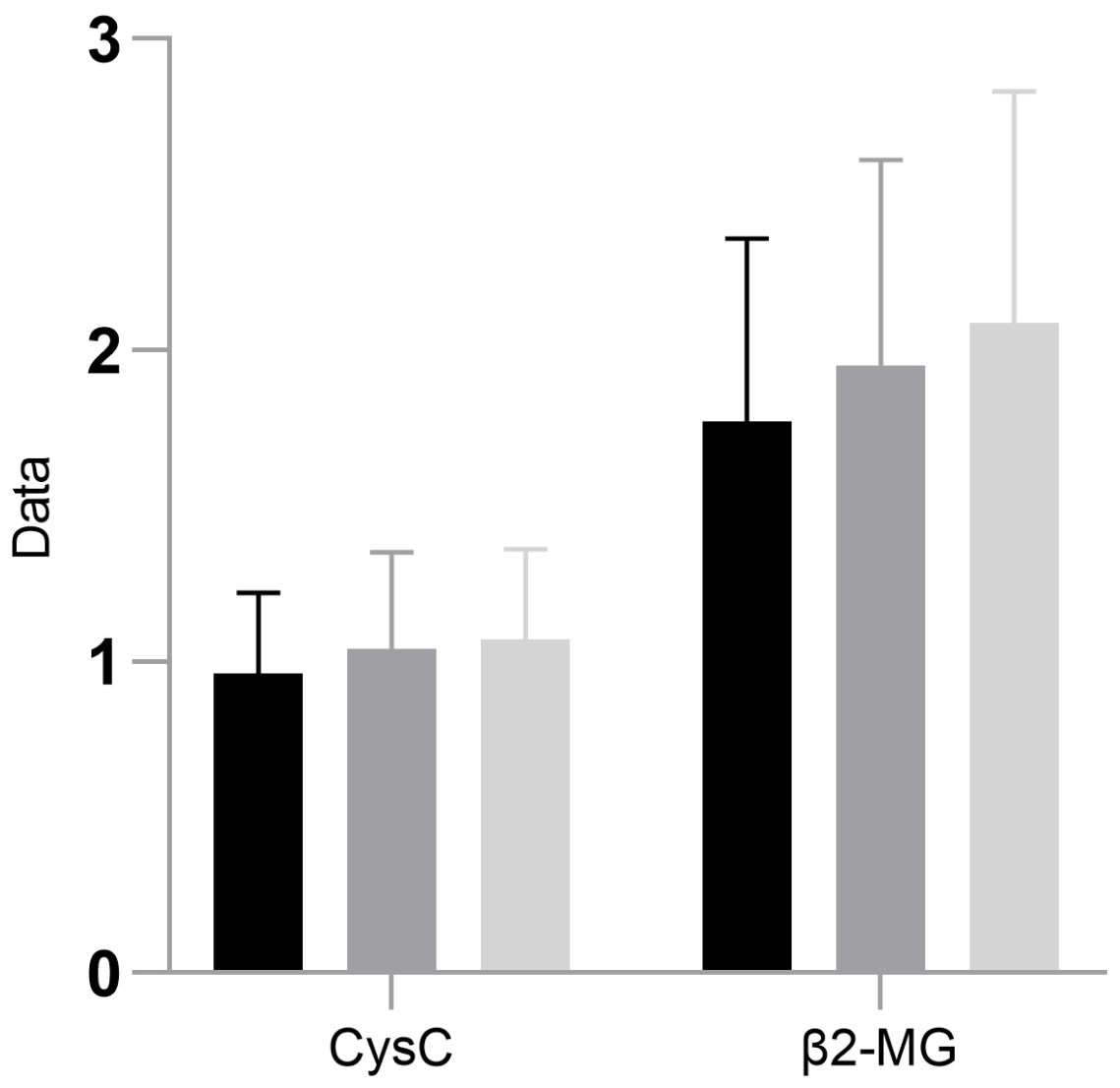

L-FAR group

M-FAR group

H-FAR group

Figure 1

Comparison of $\beta 2-M G$ and CysC levels among L-FAR, M-FAR and H-FAR groups $\beta 2-M G \otimes B l o o d ~ \beta 2$ microglobulin囚CysC邓Serum cystatin C; 


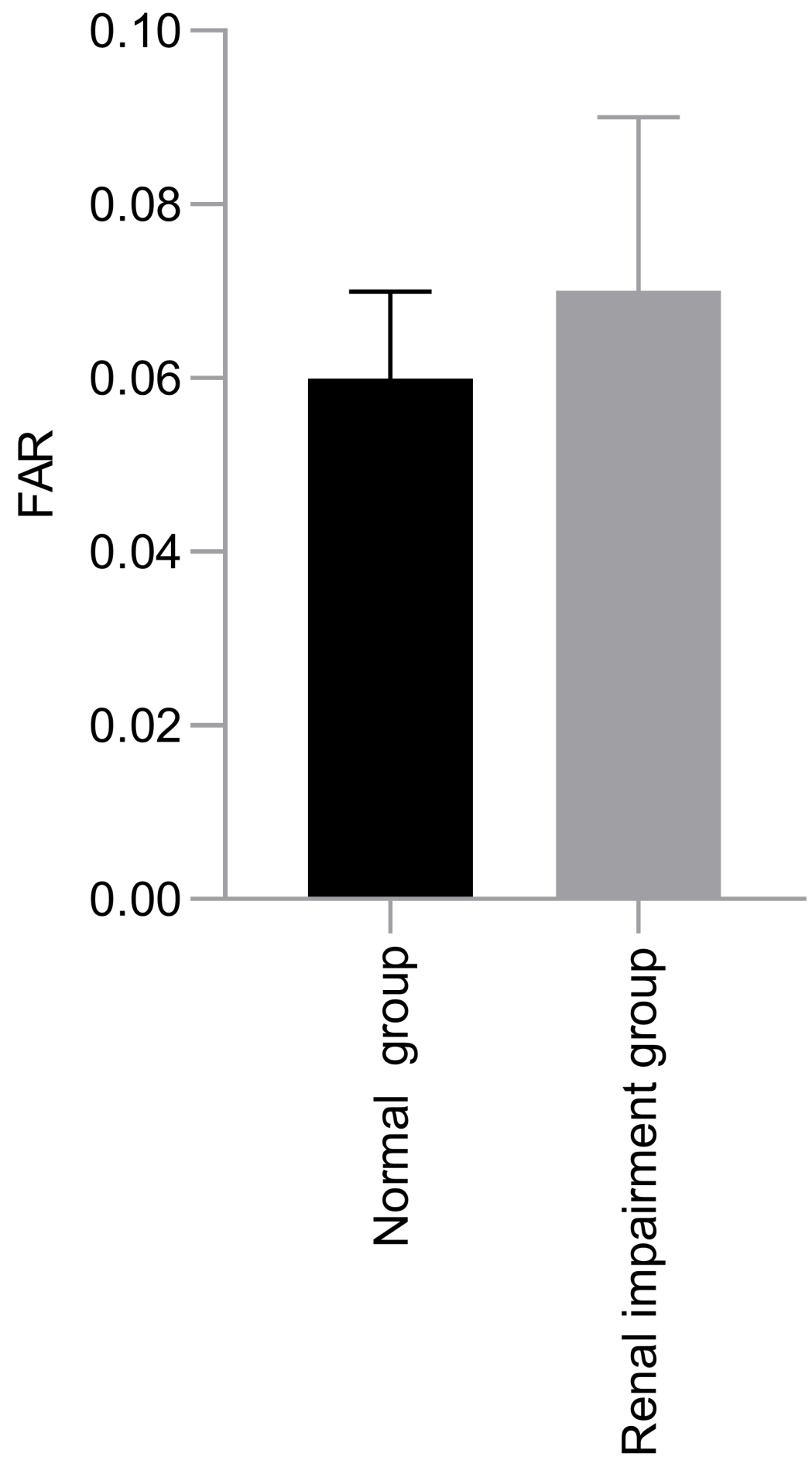

Figure 2

Comparison of FAR between normal renal function group and Renal impairment group FAR

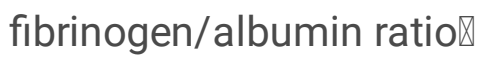

